Retrospective Evaluation

\title{
e Ultrasound Guided Peripheral Nerve Stimulation Implant for Management of Intractable Pain after Inguinal Herniorrhaphy
}

Foad Elahi, MD, Chandan G. Reddy, MD, and David Ho, MD

From: University of lowa, lowa

City, lowa

Dr. Elahi and Dr. Reddy are Assistant Professors, University of lowa, lowa City, lowa. Dr. Ho is with the Academic Scientist Trantion Program, University of lowa, lowa City, lowa.

Address Correspondence: Foad Elahi, MD Dept. of Anesthesiology University of lowa 200 Hawkins Drive, 5JPP lowa City, lowa 52242 E-mail: foad-elahi@uiowa.edu

Disclaimer: There was no external funding in the preparation of this manuscript. Conflict of interest: Each author

certifies that he or she, or a member of his or her immediate

family, has no commercial association (i.e., consultancies, stock ownership, equity interest, patent/licensing arrangements, etc.) that might pose a conflict of interest in connection with the submitted manuscript.

Manuscript received: 05-20-2014 Revised manuscript received: 08-09-2014

Accepted for publication: 09-04-2014

Free full manuscript: www.painphysicianjournal.com
Background: Inguinal hernia repair is one of the most common operations performed worldwide. Intractable pain following this operation is a potential debilitating complication. The exact etiology of this complex pain is unknown and the treatment of chronic pain after inguinal herniorrhaphy can be a difficult task for both the patient and the clinician.

Objectives: The objectives of this study are to identify the ability of peripheral nerve electrical stimulation to decrease post inguinal herniorrhaphy pain, increase patients' functionality, and decrease pain medication use.

Study Design: Three patients with intractable pain after inguinal herniorrhaphy were included in this case series. Two patients had right-sided inguinal repair and one had a leftsided repair. Pain in these patients all began after the inguinal repair and had an average pain duration of 3.4 years after surgery. All 3 patients had been treated with multiple pain management modalities without significant pain improvement. We will describe the clinical course of these patients who presented with chronic intractable pain. After a period of failed conservative medical management and repetitive successful nerve blocks, we decided to proceed with utilizing electrical nerve stimulation as a treatment modality.

Setting: This retrospective study was done at the university hospital and has an IRB assigned number.

Results: After careful consideration of the patients' history and physical examination and a thorough psychological assessment, we proceeded with a temporary percutaneous electrical neurostimulation that provided significant pain relief. Ultrasound guided permanent percutaneous electrical neurostimulation implant was shown to provide significant pain relief at 12-month follow-ups. We reviewed all existing pertinent medical literature related to the management of post herniorrhaphy pain. This case series adds to our current knowledge for chronic intractable post herniorrhaphy pain management.

Limitations: This study is a retrospective assessment of a new technique that was applied to a limited number of cases. It remains to be determined whether this technique is superior to the classical open surgical technique in the future. Our findings warrant further studies on the utilization of peripheral nerve stimulation with chronic post herniorrhaphy pain.

Key words: Peripheral nerve stimulation, ilioinguinal nerve, iliohypogastric nerve, nerve block, inguinal hernia repair, ultrasound guided procedure

Pain Physician 2015; 18:E31-E38 
nguinal hernia repair is one of the most common surgical procedures. The majority of the literature regarding hernia repair has focused on preventing a recurrence of the hernia. Thanks to the introduction of the Mesh technique, the recurrence rate has greatly declined. However, it has not been until recently that post herniorrhaphy chronic pain has come into focus. The prevalence of post herniorrhaphy pain is estimated to be as high as $54 \%$, with a cumulative prevalence of $30 \%$ at 3 years postoperatively $(1,2)$. Variables affecting post hernia repair pain include operative technique (i.e., laparoscopic vs. open), age, and pain level before the operation. Chronic pain after inguinal repair has risen over the past decade. It has a significant impact on quality of life and there is increased use of health services by patients who have chronic pain $(3,4)$. The identifiable service cost is likely to be underestimated because it does not include the societal cost of time off work and reduced performance of patients with chronic pain.

The 2 most common types of pain following herniorrhaphy are nociceptive pain and neuropathic pain. Nociceptive pain, or inflammatory pain, is due to visceral or somatic tissue damage from surgical dissection, sutures, or application of meshes. Neuropathic pain is pain that is a result of a disturbance in the nerve, which is typically a result of injury to the nerve(s). In many cases, mild neural insult will spontaneously recover during the usual postoperative period. Persistent postsurgical pain beyond the natural tissue healing process is most likely neuropathic in nature. The 2 nerves most commonly injured during and after an inguinal hernia repair are the ilioinguinal and iliohypogastric nerves.

Ilioinguinal and iliohypogastric nerve damage can occur not only during the surgical hernia repair itself, but also during the healing stage as a consequence of fibrotic entrapment of the nerves. The pain due to injury to these nerves is usually responsive to pharmacological treatment. This includes the use of the "analgesic ladder" ranging from non-steroidal antiinflammatory drugs, like ibuprofen, and membrane stabilizers, like gabapentin, to opioids. Modalities outside of pharmacotherapy include diagnostic and therapeutic peripheral nerve blocks, radiofrequency treatment, cryoanalgesia ablation, transcutaneous electrical nerve stimulation, mesh/staple removal, neurectomy, and peripheral nerve stimulation (5) (Table 1). A peripheral nerve block is a useful regional anesthesia and analgesic technique. It can be used as a diagnostic tool as well as part of the treatment plan. In post herniorrhaphy pain, one common nerve block is the ilioinguinal/ iliohypogastric block. An ultrasound-guided technique for this procedure has been described in adults (6-8). Despite the various techniques and ongoing efforts, most treatment modalities fail due to the development of peripheral and central sensitization of both primary small fiber afferents and second-order spinal neurons. Pain management under such a situation has traditionally been difficult.

Peripheral nerve stimulation has emerged recently as an effective treatment modality in chronic post herniorrhaphy pain. Patients generally had great relief from the chronic pain after the stimulation. However, most of the reported techniques have several drawbacks (9-11). First, electrodes of the stimulator are placed either immediately next to the targeted nerve through surgical exploration or empirically on the incisional scars. Both techniques require repetitive testing and awake intraoperative assessment. Second, a period of trial stimulation is often required before the efficacy of the stimulator can be reliably determined. Therefore, surgical manipulation is always needed as part of the diagnostic process.

Here we report an effective peripheral nerve stimulation procedure for managing chronic post herniorrhaphy pain. This procedure relies on ultrasound guidance to place a percutaneous lead of an electrical nerve stimulation device around the ilioinguinal/iliohy-

Table 1. Medical treatment and interventional strategies commonly used for the treatment of chronic post herniorrhaphy pain.

\begin{tabular}{|l|l||}
\hline Pharmacotherapy ( monotherapy or combined medications) & $\begin{array}{l}\text { Antiepileptics (Gabapentin, Topiramate, etc), Antidepressant (Amitriptyline, } \\
\text { Nortryptiline, etc), NSAIDs, Opioids, Topical (Lidocaine, Capsicine, etc) }\end{array}$ \\
\hline Nerve Blocks & Local Anesthetic with or without Corticosteroid \\
\hline Chemical Neurolysis & Alcohol, Phenol \\
\hline Electrical Neurolysis & $\begin{array}{l}\text { Cryo Nerve Ablation (cryoanalgesia), Radiofrequency Nerve Ablation } \\
\text { (Conventional/Cooled/ Pulse Radiofrequency) }\end{array}$ \\
\hline Electrical Neuro-Stimulation & Spinal Dorsal Column, Peripheral Nerve \\
\hline Surgical Approaches & $\begin{array}{l}\text { Neurolysis, Neurectomy, Surgical Revision/Decompression, Mesh Removal, } \\
\text { Stich Removal }\end{array}$ \\
\hline
\end{tabular}


pogastric nerves. Once stimulation of the ilioinguinal and iliohypogastric nerves hase been confirmed with significant pain control during the trial period, implantation of the peripheral nerve stimulator on these nerves would follow in the operating room in the same manner. This technique was applied to 3 patients with chronic post herniorrhaphy pain who failed multiple pain treatment modalities.

\section{Methods}

\section{Patients}

Three patients with histories of chronic pain after inguinal herniorrhaphy were included in this case series. Two patients had a right-sided inguinal repair while one had a left-sided repair. Pain in these patients started after the inguinal repair, with an average duration of 3.4 years.

The pain severity prior to the electrical stimulation ranged from 7 to 9 on the visual analogue scale (VAS). All 3 patients had been treated with pain treatment modalities without success (Table 2).

\section{CASE 1}

A 75-year-old Caucasian male has a history of right inguinal hernia repair 3 years ago. He had a surgical revision and removal of hernia mesh due to intractable pain one month after the first surgery. The second repair was done with the mesh placement on postoperative day 7 due to abdominal pain.

He was referred to the pain center with chronic persistent, lancinating, burning, and occasional sharp pain in the right lower abdomen and anterior groin. The pain had been present for 3 years since the last surgical hernia repair.

The patient rated the average pain as 9 out of 10 in severity on the VAS. He reported significant tenderness on the upper and medial thighs, and inguinal region, with occasional radiation to the genitalia. He denied numbness or tingling in his lower extremities. He noticed that his pain would aggravate with walking and prolonged standing, but pain severity remained the same at rest. A physical examination, which included a detailed sensory examination, was normal. Prior treatments included physical therapy, transcutaneous electrical nerve stimulation, nonsteroidal local anesthetic cream, morphine, and hydrocodone. He reported that none of his prior treatments gave him significant pain relief.

After careful consideration of his prior treatment and re-implementation of multimodal treatment strategies, including a pain psychologist assessment and treatment, a decision to perform an ilioinguinal/iliohypogastric nerve block was made. A trial of the nerve block provided some pain reduction with no long term benefit. At this stage we discussed a trial of right-sided iliohypogastric and ilioinguinal nerve stimulation. After an ultrasound guided peripheral nerve stimulator was placed, the patient's pain decreased by $70 \%$. He noticed that when the stimulation was turned off, the pain recurred. This result was reproducible by repetitively turning the stimulation device on and off. After a course of successful response, he underwent a permanent right-sided peripheral electrical nerve stimulator implant. We utilized 2 sets of 8 contact electrodes ( $2 \times 8$ compact, 3778; Medtronic Neuromodulation, Minneapolis, MN, USA). Neurostimulator parameters were 1.0 millivolts amplitude, 300 pulse widths, and 80 Hertz frequencies.

The procedure was done under ultrasound guidance in the operating room with the patient completely awake and cooperative. The superior lead was directed and placed over the iliohypogastric nerve and the secondary lead was aimed for the ilioinguinal nerve. Both leads were advanced and stimulated to have adequate coverage of the painful areas without painful paresthesias. The peripheral nerve stimulator implantation procedure was performed with anchoring, tunneling, and battery generator placement under local anesthetic injection and monitored anesthetic care (Fig. 1).

At his one month follow-up, he reported reduced pain at $4-5$ out of 10 on VAS. At his 3 month follow-

Table 2. Patients' data; pain score based on visual analog scale (VAS)

\begin{tabular}{|l|c|c|c|c|c|c||}
\hline Patient & Age/ Gender & Side & $\begin{array}{c}\text { Duration of } \\
\text { Pain Prior to } \\
\text { Hernia Repair }\end{array}$ & $\begin{array}{c}\text { Duration of } \\
\text { Pain Prior Nerve } \\
\text { Stimulation Implant }\end{array}$ & $\begin{array}{c}\text { Average Pain Score } \\
\text { Before and After } \\
\text { Hernia Repair }\end{array}$ & $\begin{array}{c}\text { Pain after Nerve } \\
\text { Stimulation Implant- } \\
\text { 12 Months Follow-Up }\end{array}$ \\
\hline Case-1 & 75/M & Right & 1 year & 3 years & 9 & $1-2$ \\
\hline Case-2 & 52/M & Left & 3 months & 5 years & 9 & 1 \\
\hline Case-3 & 32/M & Right & 3 months & 2 years and 4 months & 9 & $1-2$ \\
\hline
\end{tabular}




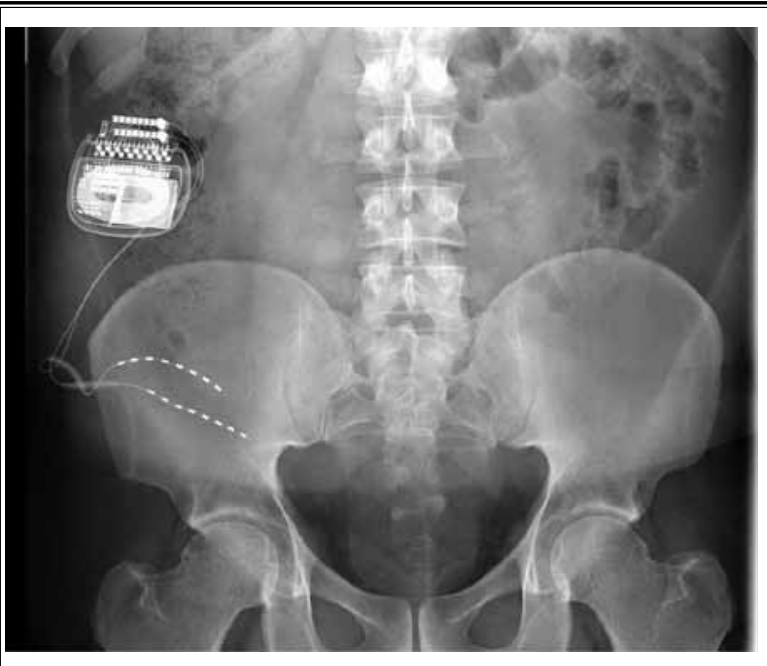

Fig. 1. Anterior posterior abdominal $x$-ray showing the electrodes with an implantable pulse generator.

up, his VAS score decreased to $0 / 10$ at rest and 1/10 with activity. He reported $100 \%$ satisfaction with pain control. He was able to wean off narcotic medication. At his one year follow-up he was taking acetaminophen over the counter for occasional discomfort. He and his wife felt that there has been a significant difference in his personal and social life engagement since the procedure.

\section{Case 2}

A 52 year-old African American male underwent left inguinal hernia repair 5 years ago. The patient had been having persistent burning and sharp pain ever since the surgery. The pain distribution was in the left groin area and was worse with any type of physical activity, including walking and prolonged standing. The pain occasionally radiated down to his leg after a short course of walking. He described pain aggravation over his left groin and testicle when he wore tight underwear or when tightening his belt.

Previous treatments included multiple ilioinguinal and iliohypogastric nerve blocks, surgical neurectomy, gabapentin, and hydrocodone. The neurectomy provided temporary relief for about 3 months but the pain had since returned. He reported daily pain severity from 8 to 10 on VAS. Physical examination, including a detailed sensory examination, was normal.

On the day of presentation, after a careful consideration of prior failed interventions, we decided to proceed with a trial period of the ultrasound guided electrical neuromodulation placement over the left ilio- hypogastric and ilioinguinal nerve. The patient reported a dramatic response of $90-100 \%$ pain relief both at rest and with activities. He was able to change positions without pain and tolerated long distance walking with minimal discomfort. He underwent a permanent peripheral nerve implant for the left iliohypogastric and ilioinguinal nerve.

We utilized 2 sets of 8 contact electrodes ( $2 \times 8 \mathrm{com}-$ pact, 3778; Medtronic Neuromodulation, Minneapolis, MN, USA). The superior lead was placed at the iliohypogastric nerve and the secondary lead was at the ilioinguinal nerve. The patient reported great pain coverage with stimulation in the operating room. Neurostimulator parameters were 1.0 millivolts amplitude, 350 pulse widths, and 80 Hertz frequencies.

On follow-up for stich removal, the patient reported complete pain relief for the first time in 5 years. He was very pleased with the result. He was able to completely wean off narcotic medication and started his new job at a local store. On his 12 month follow-up he was not taking any medication.

\section{Case 3}

A 32 year-old male who had a right-sided hernia patch repair 2 years and 4 months prior presented to the pain clinic. Subsequent to his hernia repair, he developed pain involving the right lower abdominal region, radiating down to the right side of the groin and the testicle. The pain characteristic was described as a constant burning pain with exacerbation during activities. The patient rated the pain at an average of 9 on the VAS in severity. Physical examination, including detail sensory examination, was normal. Previous treatments included nerve blocks, multimodal treatment, and significant dose of opioid medication, but no treatment had provided significant pain relief. The patient kept his full-time job during this period and reported being seen by many physicians but had not had any improvements in his symptoms.

We implemented a multimodal treatment strategy along with assessment from a pain psychologist. The patient understood the electrical neuromodulation as a modality for treatment and was willing to proceed with the right iliohypogastric and ilioinguinal nerve stimulation trial. An ultrasound guided percutaneous iliohypogastric and ilioinguinal nerve stimulation trial was performed in the operating suite under routine sterile conditions. Stimulatory frequency was applied to a comfortable level without muscle stimulation. During the stimulation trial period, the patient reported $100 \%$ 
pain relief. Because of the positive response, he underwent permanent right iliohypogastric and ilioinguinal peripheral nerve stimulator implant. The 2 leads were placed in a $\mathrm{V}$ shaped fashion over the iliohypogastric and ilioinguinal nerve. We utilized 2 sets of 8 contact electrodes (2x8 compact, 3778; Medtronic Neuromodulation, Minneapolis, MN, USA.) Neurostimulator parameters were 1.5 millivolts amplitude, 300 pulse widths, and 100 Hertz frequencies.

The patient reported to be pain-free after stimulation from both leads. At the 12 month follow-up, the patient reported minimal pain of $1-2$ on VAS. He was $100 \%$ satisfied with the result. He was kept on $300 \mathrm{mg}$ gabapentin with $25 \mathrm{mg}$ of amitriptyline on his one year follow-up.

\section{TechNique}

\section{Ultrasound Guided Ilioinguinal/ Iliohypogastric Nerve Percutaneous Electrical Nerve Stimulation Placement}

In order to describe the importance of utilizing ultrasound to safely identify the ilioinguinal/iliohypogastric nerves, here we review the ilioinguinal/iliohypogastric nerve anatomy on the anterior abdominal wall.

The general anatomy of the iliohypogastric and ilioinguinal nerves has been well documented in standard anatomy textbooks. The ilioinguinal nerve emerges from the lateral border of the psoas major just inferior to the iliohypogastric, and passes obliquely across the quadratus lumborum and iliacus. The ilioinguinal nerve then perforates the transversus abdominis near the anterior part of the iliac crest. It communicates with the iliohypogastric nerve between the transversus and the internal oblique muscle and runs in the plane between the internal oblique muscle and transversus abdominis muscles. The iliohypogastric nerve runs superior to the ilioinguinal nerve. Recent data related to common anatomical variations in these nerves are helpful for navigating the ultrasound. The ilioinguinal nerve usually enters the abdominal wall $2.8 \pm 1.1$ $\mathrm{cm}$ medial and $4.6 \pm 1.2 \mathrm{~cm}$ inferior to the anterior superior iliac spine (ASIS) and terminates $3.6 \pm 0.5 \mathrm{~cm}$ lateral to the midline. The iliohypogastric nerve enters the abdominal wall $2.8 \pm 1.3 \mathrm{~cm}$ medial and $1.4 \pm 1.2 \mathrm{~cm}$ inferior to the ASIS and terminates $4 \pm 1.3 \mathrm{~cm}$ lateral to the midline (12).

In order to identify specific nerves with ultrasound, we usually place patients in the supine position. It is extremely important to identify the anatomical land- marks on the abdominal surface, which includes the anterior superior iliac spine, rectus abdominis, umbilicus, symphysis pubis, and semilunar line (Fig. 2).

Usually a high frequency linear probe (12 megahertz) can be placed on the anterior abdominal wall along the line delineated by the ASIS and the umbilicus. This orientation will provide optimal visibility to identify the peritoneum, transverse abdominis muscle, and internal oblique muscle. In some cases the external oblique muscle is recognizable by slightly sliding the probe cephalad without changing the orientation of the probe.

The ilioinguinal and iliohypogastric nerves can be visualized as 2 round hypoechoic structures adjacent to each other with hyperechoic borders. They lie in the plane between the internal oblique muscle and the transversus abdominis muscle. This muscle plane is void of any significant vascular structure (Fig. 3). Entry point of the needle should be just a few millimeters medial to the ASIS. Entry point of the needles should be tailored to the patient's body habit.

After the initial ultrasound, skin overlying the area should be prepped and draped in a sterile fashion. Under ultrasound guidance, an entry point is selected. It should be as close as possible to the ultrasound probe and should be a few millimeters away from ASIS. A 14 gauge Touhy needle is guided into the fascial plane between the internal oblique and transversus abdominis while keeping neural structures visualized under ultrasound. When the tip of the needle is confirmed to be adjacent to the neural structure, 2 neurostimulator lead

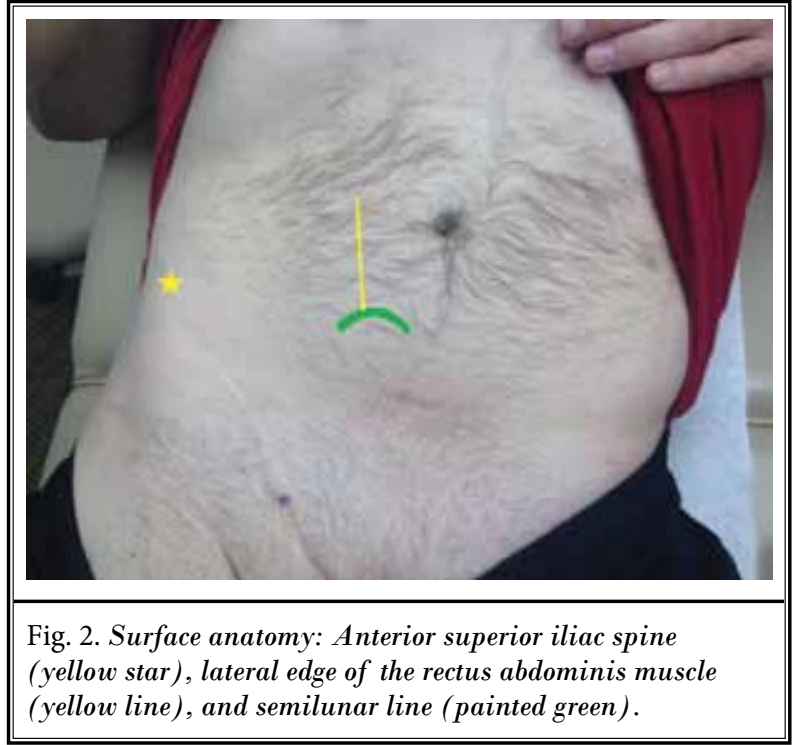




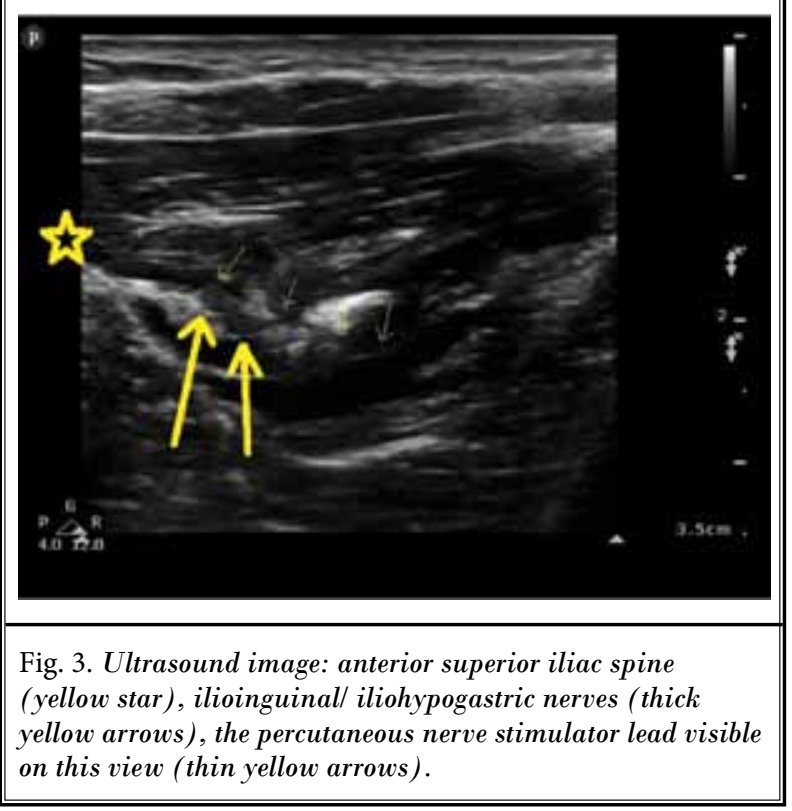

catheters are placed. The distance between the ilioinguinal and iliohypogastric nerves can easily be covered with one stimulator lead. We prefer to insert 2 leads and advance the lead as far as possible along the neural structure. The entire procedure can be performed with local anesthetic only. After both leads are advanced and stimulated to give adequate coverage of the painful areas without generating paresthesia, the leads are secured on the skin. The entire procedure is performed on an out-patient basis.

The permanent implant is exactly the same as the initial trial with the addition of securing the leads under the fascia and connecting the leads to the implantable pulse generator. The permanent implant can be done under monitored anesthesia care while the patient remains awake (Fig. 1).

\section{Literature Review}

Treatment of post herniorrhaphy pain has not been well established. Most publications on the treatment of post herniorrhaphy pain are case reports and case series.

We reviewed the entire PubMed medical literature using the following words: "pain," "peripheral," "never stimulation," "inguinal," and "post herniorrhaphy." All studies published between January 1900 and March 2014 was included. Language was restricted to English, and the reviews were checked for additional studies.

Treatment modalities reported in the literature included medication, surgical intervention, local nerve block, cryoablation, pulsed radiofrequency, and nerve stimulation.

In terms of medications, little has been objectively studied solely in the context of post herniorrhaphy pain. Gabapentin was shown to be of benefit in a small number of cases, and it may have a protective effect against chronic nerve pain if used preoperatively $(13,14)$. Surgical intervention of post herniorrhaphy pain includes surgical exploration, removal of surgical mesh, or surgical neurectomy. However, the efficacy of these interventions is also not well established. Most reports are retrospective case series with poorly defined diagnostic criteria for chronic pain.

Interventional treatment options for post herniorrhaphy pain vary greatly. Repetitive infusion of local anesthetic and clonidine for 3 weeks was successful for a year in a patient with recalcitrant ilioinguinal neuralgia (15). In a case series, cryoanalgesia ablation of the ilioinguinal nerve and genitofemoral nerve resulted in decreased pain scores and medication use (16). Pulsed radiofrequency treatment of these nerves has been shown to be beneficial in one case report and a case series $(17,18)$.

Four studies were found using peripheral nerve stimulation as the primary treatment modality for chronic pain after inguinal herniorrhaphy (Table 3).There were 3 case series with follow-up duration ranging from 6 weeks to 11 months. Patients typically underwent a 4-day trial stimulation before permanent nerve stimulation was implanted. In these reports, the patients had pain on a scale from 8 to 10 out of 10 before the implant and generally had satisfactory results with pain scores of $0-1$ out of 10 after the nerve stimulator had been implanted.

Lepski et al (19) published a small case control study showing significant reduction in pain intensity using peripheral nerve field stimulation (PNFS). Mean pain intensity was reduced from $7.2 / 10$ to $4.7 / 10(P<$ $0.001)$. When PNFS is combined with spinal cord stimulation (SCS), patients had significant reduction in pain inventory and SF36 scores at 12 month follow-ups $(P<$ 0.05) (19).

\section{Discussion}

Peripheral nerve stimulation has been reported as a potential effective treatment modality for post herniorrhaphy pain. Peripheral nerve stimulators are being used in multiple centers nationwide. It generally involves surgical exploration of the peripheral nerve and placement of the flat plate (paddle lead) multi-contact 
electrode immediately next to the targeted nerve. There have been reports of nerve injury from electrode insertion or stimulation-related fibrosis, which made peripheral nerve stimulators less desirable for both surgeons and patients. In this case series, instead of the surgical approach, ultrasound guided percutaneous electrical lead stimulation of the ilioinguinal/iliohypogastric nerves was used. Following a positive response, a permanent percutaneous peripheral nerve stimulator can then be implanted, with leads implanted as close as possible to the ilioinguinal/iliohypogastric nerves. This technique was shown to be effective in 3 patients with chronic intractable post herniorrhaphy pain.

Innervation of the inguinal region comes mostly from T12 and L1 spinal roots that form the peripheral nerve branches of ilioinguinal and iliohypogastric nerves. Chronic pain related to ilioinguinal and/or iliohypogastric nerve entrapment/damage is commonly reported following repair of an inguinal hernia.

In the management of chronic intractable pain syndromes of different etiologies, there has been a shift from destructive interventions to stimulation procedures. Electrical stimulation is used often in patients with persistent neuropathic pain syndromes.

There is a growing amount of literature to support the success of peripheral neuromodulation against neuropathic pains. Thus far, the use of peripheral nerve stimulation for neuropathic pain has been associated with consistently positive results (20).

Like paddle style electrodes, neuromodulatory effects administered through percutaneous leads can generate similar clinical benefits with less side effects.

It is important to note that muscles are often activated as the amplitude of the stimulation is increased.
It is true both during the trial and the permanent implantation. Strategies such as low energy and high frequency stimulation may be utilized as a solution.

Percutaneous placement of electrodes is a minimally invasive procedure that allows for a therapeutic trial prior to surgical placement of a permanent electrode with an implantable pulse generator.

\section{Conclusion}

Post herniorrhaphy pain and its treatment has not been well established.

Electrical neuromodulation via peripheral nerve stimulation should be reserved for highly selected cases with chronic intractable pain. A well-motivated and psychologically stable patient is a prerequisite and can increase the likelihood of success of the treatment modality of choice.

Obtaining a detailed history and physical examination is imperative, enabling doctors to make diagnoses such as scar neuroma that can be treated with less invasive treatment modalities.

Multimodal treatment strategies including pharmacotherapy, physical therapy, and biofeedback techniques should be the first choices in the chronic pain management. Ilioinguinal/iliohypogastric nerve block can provide clinical evidence for nociceptor pathways. However, pain responses to nerve blocks should be cautiously interpreted.

We incorporated the use of ultrasound guided ilioinguinal/iliohypogastric nerve stimulation placement during the trial period and for the permanent implant to eliminate the need for a surgical trial and to provide an indication for location of electrode placement.

Utilizing an ultrasound and percutaneous lead

Table 3. Peripheral nerve stimulation as a treatment modality for chronic pain after inguinal herniorrhaphy. Pain reported on visual analog scale (VAS).

\begin{tabular}{|c|c|c|c|c|c|c|}
\hline First Author & Year & Method & Study Type & $\begin{array}{l}\text { No. of } \\
\text { Cases } \\
\end{array}$ & $\begin{array}{c}\text { Pain Before } \\
\text { Implant } \\
\end{array}$ & Pain After Implant \\
\hline Stinson et al (9) & 2001 & Simulator trial followed by implant & Case series & 3 & $8-10$ & 0 after 6 weeks \\
\hline Paicius et al (10) & 2006 & Simulator trial followed by implant & Case series & 1 & 10 & $0-1$ after 11 months \\
\hline $\begin{array}{l}\text { Rauchwerger et al } \\
\text { (11) }\end{array}$ & 2008 & Simulator trial followed by implant & Case series & 3 & $8-9$ & $\begin{array}{c}\text { Satisfactory after } 6 \\
\text { months }\end{array}$ \\
\hline Lepski et al (19) & 2013 & $\begin{array}{l}\text { Combined spinal cord (SCS) and } \\
\text { peripheral nerve field stimulation } \\
\text { (PNFS) }\end{array}$ & Case-control & $\begin{array}{c}4 \text { cases, } 36 \\
\text { controls }\end{array}$ & 7.2 & $\begin{array}{l}\text { Average pain for } 3 \text { days: } \\
4.7 \text { for PNFS, } 3.4 \text { for } \\
\text { SCS, } 2.4 \text { for combined } \\
\text { stimulation }\end{array}$ \\
\hline $\begin{array}{l}\text { Elahi - Current } \\
\text { Study }\end{array}$ & 2014 & $\begin{array}{l}\text { Ultrasound Guided Percutaneous } \\
\text { Ilioinguinal / Iliohypogastric Nerve } \\
\text { Stimulator Implant }\end{array}$ & Case Series & 3 & $7-10$ & $\begin{array}{l}90 \% \text { satisfaction rate } \\
\text { with reported pain } 1 \text {, at } \\
12 \text { months follow-up }\end{array}$ \\
\hline
\end{tabular}


placement approach causes none to negligible intra- and postoperative discomfort at the site of the electrodes.

Little postoperative pain and satisfactory response to the procedure itself lead us to conclude that this technique can be considered a very useful alternative to the classical operative procedure. It remains to be determined whether this technique is indeed superior to the classical open surgical technique in the future.

Patients reported significant relief at long-term follow-ups.

Randomized controlled trials are greatly needed to confirm findings in these reports.

\section{References}

1. Poobalan AS, Bruce J, Smith WC, King PM, Krukowski ZH, Chambers WA. A review of chronic pain after inguinal herniorrhaphy. Clin J of Pain 2003; 19:48-54.

2. Poobalan AS, Bruce J, King PM, Chambers WA, Krukowski ZH, Smith WC. Chronic pain and quality of life following open inguinal hernia repair. $\mathrm{Br}$ ] Surgery 2001; 88:1122-1126.

3. Wantz, G. Testicular atrophy and chronic residual neuralgia as risks of inguinal hernioplasty. The Surgical Clinics of North America 1993; 73:571-581.

4. Becker N, Bondegaard Thomsen A, Olsen AK, Sjøgren P, Bech P, Eriksen J. Pain epidemiology and health related quality of life in chronic non-malignant pain patients referred to a Danish multidisciplinary pain center. Pain 1997; 73:393-400.

5. Thomassen I, van Suijlekom $H$, van der Gaag A, Nienhuijs S. Intervention techniques for chronic postherniorrhaphy pain. European Surgery 2012; 44:132-137.

6. Gofeld M, Christakis M. Sonographically guided ilioinguinal nerve block. Journal of Ultrasound in Medicine 2006; 25:1571-1575.

7. Eichenberger $U$, Greher $M$, Kirchmair L, Curatolo M, Moriggl B. Ultrasoundguided blocks of the ilioinguinal and iliohypogastric nerve: Accuracy of a selective new technique confirmed by ana- tomical dissection. Br J Anaesthes 2006; 97:238-243.

8. Thomassen I, van Suijlekom J, van de Gaag A, Ponten J, Nienhuijs S. Ultrasound-guided ilioinguinal/iliohypogastric nerve blocks for chronic pain after inguinal hernia repair. Hernia 2013; 17: 329-332.

9. Stinson LW, Roderer GT, Cross NE, Davis BE. Peripheral subcutaneous electrostimulation for control of intractable post-operative inguinal pain: A case report series. Neuromodulation 2001; 4:99-104.

10. Paicius RM, Bernstein CA, Lempert-Cohen C. Peripheral nerve field stimulation in chronic abdominal pain. Pain Physician 2006; 9:261-262.

11. Rauchwerger JJ, Giordano J, Rozen D, Kent JL, Greenspan J, Closson CW. On the therapeutic viability of peripheral nerve stimulation for ilioinguinal neuralgia: Putative mechanisms and possible utility. Pain Practice 2008; 8:138-143.

12. Klaassen Z, Marshall E, Tubbs RS, Louis RG Jr, Wartmann CT, Loukas M. Anatomy of the ilioinguinal and iliohypogastric nerves with observations of their spinal nerve contributions. Clin Anat 2011; 24:454-461.

13. Benito-León J, Picardo A, Garrido A, Cuberes R. Gabapentin therapy for genitofemoral and ilioinguinal neuralgia. Jour- nal of Neurology 2001; 248:907-908.

14. Sen H, Sizlan A, Yanarate $O$, Senol MG, Inangil $G$. The effects of gabapentin on acute and chronic pain after inguinal herniorrhaphy. European Journal of Anaesthesiology 2009; 26:772-776.

15. Maaliki H, Naja Z, Zeidan A. Repeated ilioinguinal block using a catheter technique for pain relief in inguinal neuralgia. Pain Practice 2008; 8:144-146.

16. Fanelli R, DiSiena M, Lui F, Gersin K. Cryoanalgesic ablation for the treatment of chronic postherniorrhaphy neuropathic pain. Surgical Endoscopy and Other Interventional Techniques 2003; 17:196-200.

17. Mitra R, Zeighami A, Mackey S. Pulsed radiofrequency for the treatment of chronic ilioinguinal neuropathy. Hernia 2007; 11:369-371.

18. Cohen SP, Foster A. Pulsed radiofrequency as a treatment for groin pain and orchialgia. Urology 2003; 61:645.

19. Lepski G, Vahedi P, Tatagiba MS, Morgalla M. Combined spinal cord and peripheral nerve field stimulation for persistent post-herniorrhaphy pain. Neuromodulation: 2013; 16:84-89.

20. Slavin KV. Peripheral nerve stimulation for neuropathic pain. Neurotherapeutics 2008; 5:100-106. 